\title{
Strongly Multiplicative Labeling for Some Cycle Related Graphs
}

\author{
S K Vaidya (Corresponding author) \\ Department of Mathematics, Saurashtra University, Rajkot 360 005, Gujarat, India \\ E-mail: samirkvaidya@yahoo.co.in
}

K K Kanani

Mathematics Department, L E College, Morbi 363 642, Gujarat, India

E-mail:kananikkk@yahoo.co.in

\begin{abstract}
In this paper we discuss strongly multiplicative labeling of some cycle related graphs. We prove that cycle with one chord, cycle with twin chords and cycle with triangle are strongly multiplicative graphs. In addition to this we prove that the graphs obtained by fusion and duplication of vertices in cycle admit strongly multiplicative labeling.
\end{abstract}

Keywords: Strongly multiplicative labeling, Strongly multiplicative graph, Cycle

\section{Introduction}

We begin with simple, finite, connected and undirected graph $G=(V(G), E(G))$. Here elements of sets $V(G)$ and $E(G)$ are known as vertices and edges respectively. In the present work $C_{n}$ denotes cycle with $n$ vertices and $N(v)$ denotes the set of all neighboring vertices of $v$. For all other terminology and notations we follow (Harary F., 1972). We will give brief summary of definitions which are useful for the present investigations.

Definition 1.1 A chord of a cycle $C_{n}$ is an edge joining two non-adjacent vertices of cycle $C_{n}$.

Definition 1.2 Two chords of a cycle are twin chords (consecutive chords) if they form a triangle with an edge of the cycle $C_{n}$.

For positive integers $n$ and $p$ with $3 \leq p \leq n-2, C_{n, p}$ is the graph consisting of a cycle $C_{n}$ with a pair of twin chords with which the edges of $C_{n}$ form cycles $C_{p}, C_{3}$ and $C_{n+l-p}$ without chords.

Definition 1.3 Cycle with triangle is a cycle with three chords which by themselves form a triangle.

For positive integers $p, q, r$ and $n \geq 6$, with $p+q+r+3=n$ then $C_{n}(p, q, r)$ denote a cycle with triangle whose edges form the edges of cycles $C_{p+2}, C_{q+2}$ and $C_{r+2}$ without chords.

Definition 1.4 If the vertices of the graph are assigned values subject to certain conditions is known as graph labeling.

For detailed survey on graph labeling we refer to 'A Dynamic Survey of Graph Labeling' by (Gallian, J., 2009).

Definition 1.5 A graph $G=(V(G), E(G))$ with $p$ vertices is said to be multiplicative if the vertices of $G$ can be labeled with distinct positive integers such that label induced on the edges by the product of labels of end vertices are all distinct.

Multiplicative labeling was introduced by Beineke and Hegde (Beineke L., 2001, p. 63-75). In the same paper they proved that every graph $\mathrm{G}$ admits multiplicative labeling and defined strongly multiplicative labeling as follows.

Definition 1.6 A graph $G=(V(G), E(G))$ with $p$ vertices is said to be strongly multiplicative if the vertices of $G$ can be labeled with $p$ distinct integers $1,2, \ldots, p$ such that label induced on the edges by the product of labels of the end vertices are all distinct.

There are three types of problems that can be considered in the above context.

(i) Construct new families of strongly multiplicative graphs;

(ii) How strongly multiplicative labeling is affected under various graph operations;

(iii) Given a graph theoretic property $\mathrm{P}$ characterize the class of graphs with property $\mathrm{P}$ that are strongly multiplicative.

In (Beineke L., 2001, p. 63-75) authors have extensively studied some problems of the types (i) and (iii) and they proved that 
- Every cycle $C_{n}$ is strongly multiplicative.

- Every wheel $W_{n}$ is strongly multiplicative.

- Complete graph $K_{n}$ is strongly multiplicative if and only if $n \leq 5$.

- Complete bipartite graph $K_{n, n}$ is strongly multiplicative if and only if $n \leq 4$.

- Every spanning subgraph of a strongly multiplicative graph is strongly multiplicative.

- Every graph is an induced subgraph of a strongly multiplicative graph.

The present work is intended to discuss problems of the types (i) and (ii). Here we investigate three new families of strongly multiplicative graphs and strongly multiplicative labeling is discussed in the context of graph operations namely duplication and fusion of vertices in cycle $C_{n}$.

Definition 1.7 Let $u$ and $v$ be two distinct vertices of a graph $G$. A new graph $G_{1}$ is constructed by fusing (identifying) two vertices $u$ and $v$ by a single vertex $x$ is such that every edge which was incident with either $u$ or $v$ in $G$ is now incident with $x$ in $G_{1}$.

Definition 1.8 Duplication of a vertex $v_{k}$ of graph $G$ produces a new graph $G_{l}$ by adding a vertex $v_{k}^{\prime}$ with $N\left(v_{k}^{\prime}\right)=N\left(v_{k}\right)$.

In other words a vertex $v_{k}{ }^{\prime}$ is said to be duplication of $v_{k}$ if all the vertices which are adjacent to $v_{k}$ are now adjacent to $v_{k}^{\prime}$ also.

\section{Main Results}

Theorem 2.1 Every cycle with one chord is strongly multiplicative.

Proof: Let $\mathrm{G}$ be the cycle $C_{n}$ with one chord and $v_{1}, v_{2}, \ldots, v_{n}$ be the consecutive vertices of $C_{n}$ arranged in the clockwise direction. Let $e=v_{1} v_{i}$ be the chord of cycle $C_{n}$.

Let us define labeling $f: V(G) \rightarrow\{1,2, \ldots, n\}$ as follows.

$f\left(v_{1}\right)=1$

$f\left(v_{i}\right) \quad=p_{I}$ where $p_{I}$ is the highest prime number such that $p_{1} \leq n$.

$f\left(v_{n}\right) \quad=p_{2}$ where $p_{2}$ is the second highest prime number such that $1<p_{2}<p_{I} \leq n$.

Now label the remaining vertices starting from $\mathrm{v}_{2}$ consecutively in clockwise direction from the set $\{1,2, \ldots, n\}$ except $1, p_{1}$ and $p_{2}$ as these numbers are already used as labels.

Then $f$ is a strongly multiplicative labeling for $G$. That is, $G$ is a strongly multiplicative graph.

Illustration 2.2 Consider a cycle $C_{6}$ with one chord. The labeling is as shown in Fig 1 .

Theorem 2.3 Every cycle with twin chords is strongly multiplicative.

Proof: Let $G$ be the cycle $C_{n}$ with twin chords. Let $v_{l}, v_{2}, \ldots, v_{n}$ be the consecutive vertices of $C_{n}$ arranged in the clock-wise direction. Let $e_{1}=v_{1} v_{i}, e_{2}=v_{1} v_{i+1}$ be two chords of $C_{n}$.

Let us define labeling $f: V(G) \rightarrow\{1,2, \ldots, n\}$ as follows.

$f\left(v_{l}\right)=1$

$f\left(v_{i}\right) \quad=p_{I}$ where $p_{I}$ is the highest prime number such that $p_{I} \leq n$

$f\left(v_{i+1}\right)=p_{2}$ where $p_{2}$ is the second highest prime number such that $1<p_{2}<p_{1} \leq n$

$f\left(v_{n}\right) \quad=p_{3}$ where $p_{3}$ is the third highest prime number such that $1<p_{3}<p_{2}<p_{1} \leq n$

Now label the remaining vertices starting from $v_{2}$ consecutively in clockwise direction from the set

$\{1,2, \ldots, n\}-\left\{1, p_{1}, p_{2}, p_{3}\right\}$.

Then the graph $G$ under consideration admits strongly multiplicative labeling. That is, every cycle with twin chords is strongly multiplicative

Illustration 2.4 Consider a cycle $C_{7}$ with twin chords. The labeling is as shown in Fig 2.

Theorem 2.5 Every cycle with triangle $C_{n}(1,1, n-5)$ is strongly multiplicative. That is

Proof: Let $G$ be the cycle $C_{n}$ with triangle. Let $e_{1}, e_{2}$ and $e_{3}$ be three chords of cycle $C_{n}$. Let $v_{1}$ and $v_{3}$ be the end vertices of $e_{1}$. Let $v_{3}$ and $v_{n-1}$ be the end vertices of $e_{2}$. Let $v_{n-1}$ and $v_{1}$ be the end vertices of $e_{3}$.

To define labeling $f: V(G) \rightarrow\{1,2, \ldots, n\}$ we will consider following cases. 
Case 1: If $n$ is odd

In this case we define labeling as

$f\left(v_{i}\right)=2 i-1 ; 1 \leq i \leq(n+1) / 2$

$$
=2(n-i+1) ;(n+1) / 2<i \leq n
$$

Case 2: If $n$ is even

In this case we define labeling as

$f\left(v_{i}\right)=2 i-1 ; 1 \leq i \leq n / 2$

$$
=2(n-i+1) ; \quad n / 2<i \leq n
$$

In each case described above the graph $G$ under consideration admits strongly multiplicative labeling. That is, cycle with triangle $C_{n}(1,1, n-5)$ is strongly multiplicative.

Remark 2.6 In above Theorem 2.5 we discuss strongly multiplicative labeling for $C_{n}(1,1, n-5)$ but it is also possible to develop strongly multiplicative labeling when three chords make all possible triangle in accordance with Definition 1.3. For the sake of brevity this discussion is not reported here.

Illustration 2.7 Consider a cycle $C_{10}$ with triangle. The labeling is as shown in Fig 3.

Theorem 2.8 The graph obtained by duplication of an arbitrary vertex in cycle $C_{n}$ admits strongly multiplicative labeling.

Proof: Let $C_{n}$ be the cycle with $n$ vertices. Let $v_{k}$ be the vertex of cycle $C_{n}$ and $v_{k}^{\prime}$ is duplication and $G$ be the resultant graph. We define labeling $f: V(G) \rightarrow\{1,2, \ldots, n+1\}$ as follows.

Case 1: $n$ is odd.

In this case we define labeling $f$ as

$f\left(v_{k}{ }^{\prime}\right)=1$

For $1 \leq i \leq n-k+1$

$$
\begin{array}{r}
f\left(v_{k+i-1}\right) \quad=2 i+1 ; 1 \leq i \leq(n-1) / 2 \\
=2(n-i+1) ;(n-1) / 2<i \leq n .
\end{array}
$$

For $n-k+2 \leq i \leq n$

$$
\begin{aligned}
& f\left(v_{k+i-n-1}\right) \quad=2 i+1 ; 1 \leq i \leq(n-1) / 2 \\
&=2(n-i+1) ; \quad(n-1) / 2<i \leq n .
\end{aligned}
$$

Case 2: $n$ is even.

In this case we define labeling $f$ as

$f\left(v_{k}^{\prime}\right)=1$

For $1 \leq i \leq n-k+1$

$$
\begin{array}{r}
f\left(v_{k+i-1}\right) \quad=2 i+1 ; 1 \leq i \leq n / 2 \\
=2(n-i+1) ; \quad n / 2<i \leq n .
\end{array}
$$

For $n-k+2 \leq i \leq n$

$f\left(v_{k+i-n-1}\right)=2 i+1 ; 1 \leq i \leq n / 2$

$$
=2(n-i+1) ; n / 2<i \leq n .
$$

In each case described above the graph $G$ under consideration admits strongly multiplicative labeling. That is, the graph obtained by duplication of an arbitrary vertex in cycle $C_{n}$ admits strongly multiplicative labeling.

Illustration 2.9 Consider a graph $G$ obtained by duplicating a vertex $v_{l}$ in cycle $C_{6}$. The labeling is as shown in Fig 4.

Theorem 2.10 The graph resulted from fusion of two vertices $v_{i}$ and $v_{j}$ (where $d\left(v_{i}, v_{j}\right) \geq 3$ ) in cycle $C_{n}$ admits strongly multiplicative labeling.

Proof: Let $C_{n}$ be the cycle with vertices $v_{l}, v_{2}, \ldots, v_{n}$. Let the vertex $v_{l}$ be fused with $v_{m}$ where $m \leq\lceil n / 2\rceil$. Let $G$ be the graph obtained by fusing $v_{l}$ and $v_{m}$. It is obvious that fusion of two vertices in cycle $C_{n}$ produces a connected graph which includes two edge-disjoint cycles $C_{m-1}$ and $C_{n-m+1}$. 
We define labeling $f: V(G) \rightarrow\{1,2, \ldots, n-1\}$ as follows.

Case 1: $n$ is odd.

Subcase I: $m-1$ is an even number and $n-m+1$ is an odd number.

In this case we define labeling $f$ as

$f\left(v_{l}\right)=m-2$

$$
\begin{aligned}
f\left(v_{i}\right) \quad=m & -2 i ; 1<i \leq(m-1) / 2 \\
& =2 i-m+1 ; \quad(m-1) / 2<i \leq m-1 \\
& =2 i-m-2 ; m<i \leq(m+n) / 2 \\
& =2 n-2 i+m+1 ;(m+n) / 2<i \leq n
\end{aligned}
$$

Subcase II: $m-1$ is an odd number and $n-m+1$ is an even number.

In this case we define labeling $f$ as

$$
\begin{aligned}
& f\left(v_{l}\right)=m-1 \\
& f\left(v_{i}\right)=m-2 i+1 ; 1<i \leq m / 2 \\
& =2 i-m ; m / 2<i \leq m-1 \\
& =2 i-m-1 ; m+1 \leq i \leq(n+m-1) / 2 \\
& =2 n-2 i+m ;(n+m-1) / 2<i \leq n
\end{aligned}
$$

Case 2: $n$ is even.

Subcase I: $m-1$ and $n-m+1$ both are odd numbers.

In this case we define labeling $f$ as

$$
\begin{aligned}
& f\left(v_{l}\right)=m-1 \\
& f\left(v_{i}\right)=m-2 i+1 ; 1<i \leq m / 2 \\
& =2 i-m ; \quad m / 2<i \leq m-1 \\
& =2 i-m-1 ; m<i \leq(n+m) / 2 \\
& =2 n-2 i+m ;(n+m) / 2<i \leq n
\end{aligned}
$$

Subcase II: $m-1$ and $n-m+1$ both are even numbers.

In this case we define labeling $f$ as

$$
\begin{aligned}
& f\left(v_{l}\right)=m-2 \\
& \begin{aligned}
f\left(v_{i}\right)=m- & 2 i ; 1<i \leq(m-1) / 2 \\
= & 2 i-m+1 ; \quad(m-1) / 2<i \leq m-1 \\
= & 2 i-m-2 ; m<i \leq(n+m+1) / 2 \\
= & 2 n-2 i+m+1 ; \quad(n+m+1) / 2<i \leq n .
\end{aligned}
\end{aligned}
$$

In each possibility described above the graph $G$ under consideration admits strongly multiplicative labeling.

That is, the graph resulted from fusion of two vertices $v_{i}$ and $v_{j}$ (where $d\left(v_{i}, v_{j}\right) \geq 3$ ) in cycle $C_{n}$ admits strongly multiplicative labeling.

Remark 2.11

(i) when $m>\lceil n / 2\rceil$ the fusion of two vertices will repeat all the graphs which are already considered earlier.

(ii) when $d\left(v_{i}, v_{j}\right)<3$ then fusion yields a graph which is not simple and it is not desirable for strongly multiplicative labeling.

Illustration 2.12 Consider a graph $G$ obtained by fusing vertex $v_{l}$ with $v_{6}$ in the cycle $C_{11}$. The labeling is as shown in Fig 5.

\section{Concluding Remarks}

On account of its diversified applications, labeled graph is the topic of contemporary interest. We have derived five new results on strongly multiplicative labeling. It is possible to investigate similar results for other graph families. There is a scope to derive analogous results in the context of different graph labeling techniques. 


\section{References}

Beineke, L. W. and Hegde, S. M. (2001). Strongly Multiplicative Graphs, Discuss. Math. Graph Theory, 21, 63-75.

Gallian, J. A. (2009). A dynamic survey of graph labeling, The Electronic Journal of Combinatorics, 16, \#DS 6. Harary, F. (1972). Graph Theory, Massachusetts, Addison Wesley.

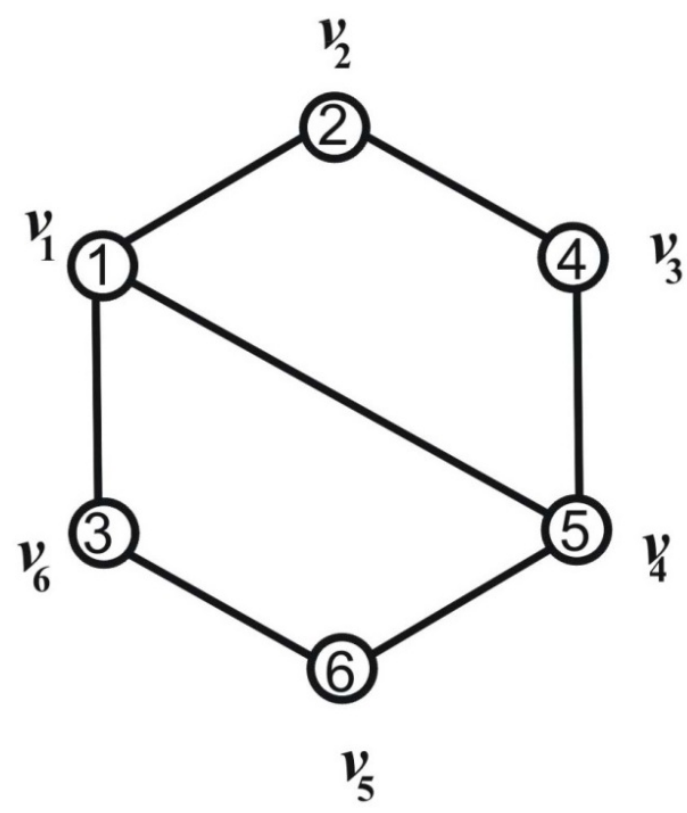

Figure 1. Cycle $\mathrm{C}_{6}$ with one chord and its strongly multiplicative labeling

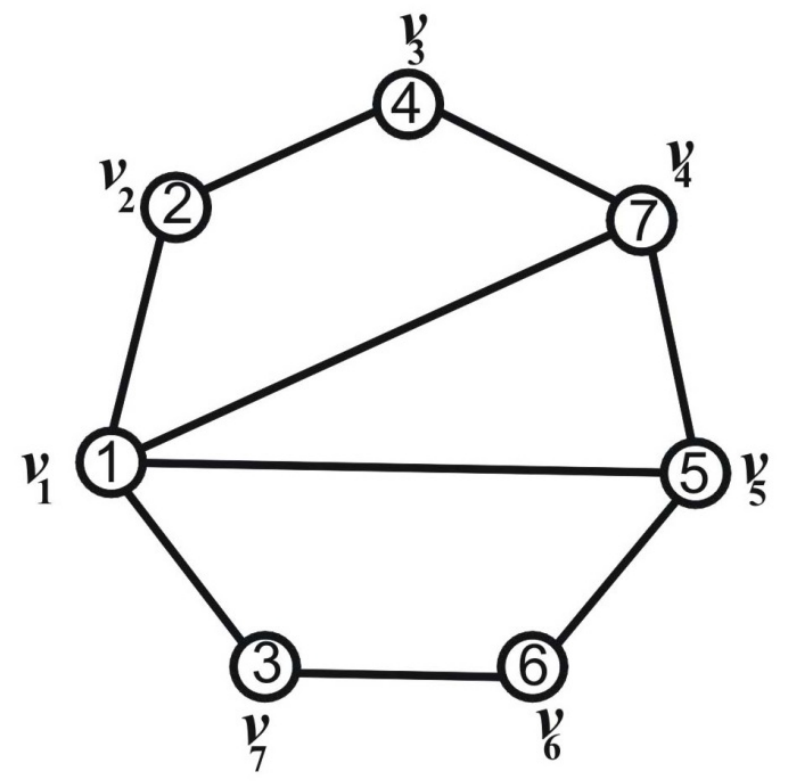

Figure 2. Cycle $\mathrm{C}_{7}$ with twin chords and its strongly multiplicative labeling 


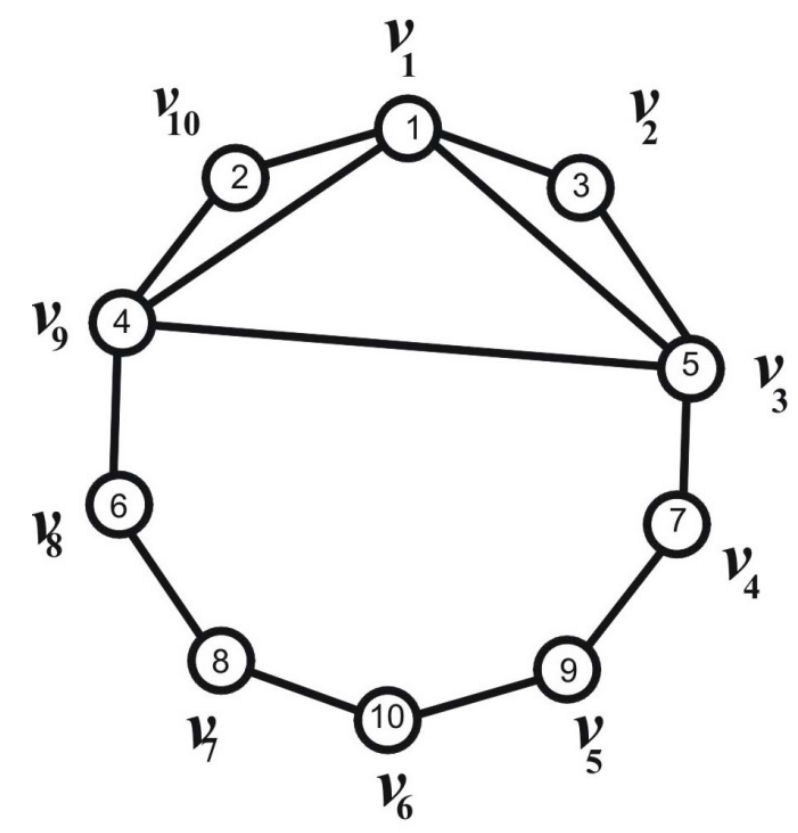

Figure 3. Cycle $\mathrm{C}_{10}$ with triangle and its strongly multiplicative labeling

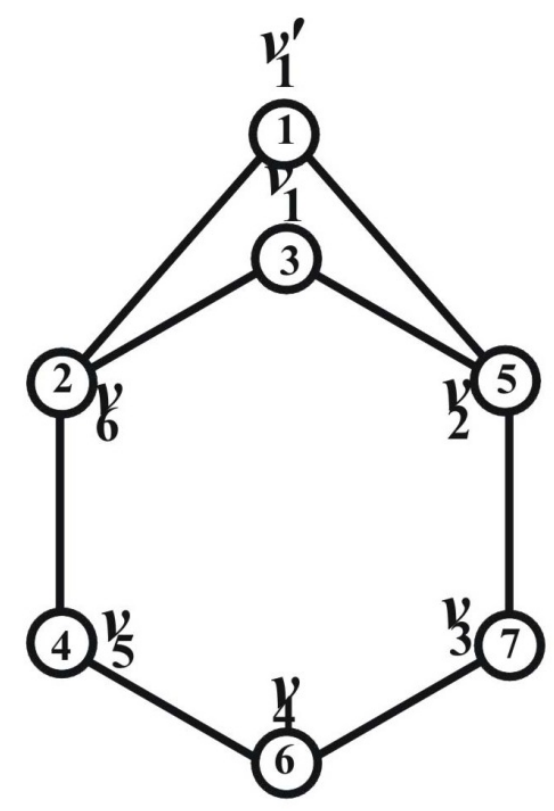

Figure 4. Duplication of a vertex $\mathrm{v}_{1}$ in Cycle $\mathrm{C}_{6}$ and its strongly multiplicative labeling 


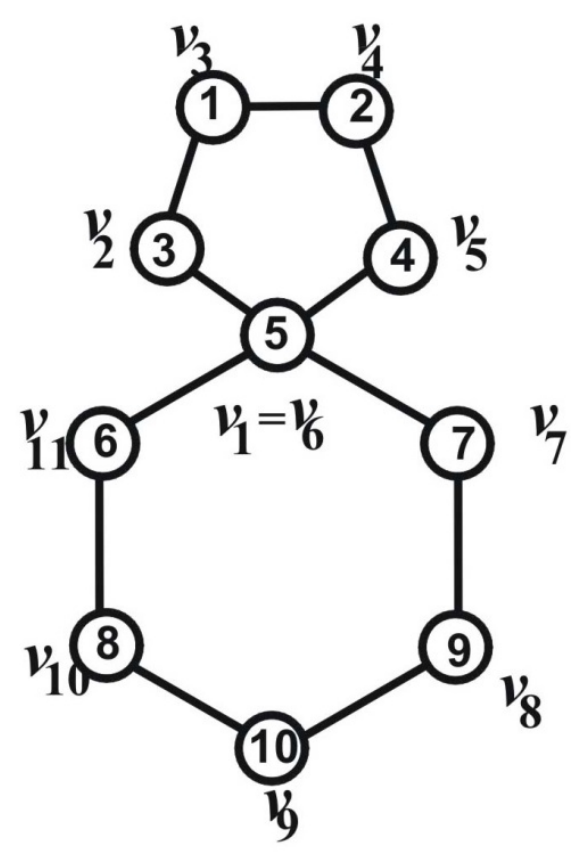

Figure 5. Fusion of $\quad \mathrm{v}_{1} \quad$ with $\mathrm{v}_{6}$ in Cycle $\mathrm{C}_{11}$ and its strongly multiplicative labeling 